\title{
Influence of the accommodation coefficient on nonlinear bubble oscillations
}

\author{
Daniel Fuster ${ }^{\mathrm{a})}$ \\ Division of Engineering and Applied Science, California Institute of Technology, Pasadena, California \\ 91125 \\ Guillermo Hauke and Cesar Dopazo \\ Laboratorio de Investigacion en Tecnologias de la Combustion-Area de Mecanica de Fluidos, Centro \\ Politecnico Superior, C/Maria de Luna 3, 50018 Zaragoza, Spain
}

(Received 22 July 2009; revised 15 April 2010; accepted 15 April 2010)

\begin{abstract}
This paper numerically investigates the effect of mass transfer processes on spherical single bubble dynamics using the Hertz-Langmuir-Knudsen approximation for the mass flux across the interface. Bubble behavior, with and without mass transfer, is studied for different values of pressure wave amplitude and frequency, as well as initial bubble radius. Whereas mass transfer processes do not seem to play a significant role on the bubble response for pressure amplitudes smaller than $0.9 \mathrm{~atm}$, they appear to have an important effect when the amplitude is greater than or equal to $1 \mathrm{~atm}$. For the later case, where the minimum liquid pressure reaches values around its vapor pressure, the importance of mass transfer depends on frequency. For frequencies in the $10^{3}-10^{5} \mathrm{~Hz}$ range and initial bubble radii of the order of tens of microns, bubble implosions with and with no mass transfer are significantly different; smaller radii display a lower sensitivity. In this regime, accurate model predictions must, therefore, carefully select the correct value of the accommodation coefficient. For frequencies greater than $10^{5} \mathrm{~Hz}$, as a first approximation mass transfer can be ignored.
\end{abstract}

(C) 2010 Acoustical Society of America. [DOI: 10.1121/1.3436520]

PACS number(s): 43.25.Yw [NAG]

Pages: 5-10

\section{INTRODUCTION}

The influence of mass transfer on bubble dynamics has been extensively studied. Different applications involving bubble oscillations have motivated the development of a variety of models accounting for evaporation and condensation processes. For small enough oscillation amplitudes, the effect of the mass transfer on the bubble behavior can be analytically determined via linear theory. For instance, Hao and Prosperetti ${ }^{1}$ have numerically studied the validity of asymptotic theory predictions for oscillating vapor bubbles.

However, the transient mass transfer analysis for Single Bubble SonoLuminiscence (SBSL) becomes more intricate due to the nonlinearity of the system model equations. Several models have been proposed in the literature taking into account mass transfer processes. A detailed comparison among them can be found in Preston et al., ${ }^{2}$ where a new reduced-order model is proposed.

A complete review of the models taking into account mass transfer processes can be found in Ref. 3. Toegel et al. ${ }^{4}$ have shown the importance of the vapor fed into the bubble along its expansion in order to explain why the sonoluminiscence upscaling theory, proposed by Hilgenfeldt et al., ${ }^{5}$ is not applicable at low frequencies. Toegel et al. ${ }^{6}$ and Hilgenfeldt et al. ${ }^{7}$ have later included mass and heat transfer across the interface in their models in order to compute the stability regions in SBSL. More complicated models, including mass

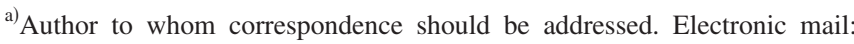
fuster@caltech.edu
}

transfer and chemical reactions, have been also developed aiming at correctly predicting the production of free radicals in sonicated liquids, ${ }^{8,9}$ as well as the degradation rates of organic compounds. ${ }^{10}$ The coupling between chemical reactions and mass transfer has been shown to be essential, for example, to predict the appearance of internal pressure waves inside the bubble under strong implosions. ${ }^{11,12}$ An et $a l .{ }^{13}$ have demonstrated the sensitivity of the appearance of internal pressure waves to the mass transfer model, even in the absence of chemical reactions. Storey and Szeri ${ }^{9}$ have shown that the condensation velocity during the collapse is determined by the diffusion rate of vapor inside the bubble; this explains why vapor is trapped within the bubble as it collapses.

In most of the previous works, it has been typically assumed that the bubble is initially composed of some undissolved gas trapped in the liquid. Steady and quasi-steady state models have widely used the hypothesis that the gas phase is in mechanical and thermodynamic equilibrium with the surrounding liquid; however, its applicability to cavitation processes is restricted, in the most favorable case, to the expansion stage. Thus, non-equilibrium conditions have been normally considered. ${ }^{14,9,4,15}$ One of the most accepted models employs an approximation for the mass flux derived from the kinetic theory of gases; an empirical accommodation coefficient is introduced to quantify the efficiency of the molecules adhering-to/abandoning the interface. ${ }^{16,17}$

Few reliable experimental data are available $e^{18,19}$ and they are limited to very restrictive conditions. ${ }^{20-23}$ As a consequence, model validation and calibration under all possible 
conditions are a cumbersome task. The uncertainty of model results introduced by the accommodation coefficient is bounded by two limiting cases: Zero mass transfer $(\beta=0)$ and equilibrium conditions. For situations between both limiting cases, the correct value of the accommodation coefficient is presumably crucial to accurately predict the bubble behavior. In fact, the sensitivity of the bubble radius evolution to the accommodation coefficient has been proposed as a method to determine its value. ${ }^{24}$

Storey and Szeri ${ }^{9}$ have shown that the condensation flux during the bubble implosion is controlled by the diffusion of vapor toward the interface. This flux depends on the bubble density, the diffusion coefficient and the concentration gradient, which is determined by the vapor trapped inside the bubble and the conditions at the interface. If the characteristic bubble implosion time scales are much smaller than those of mass transfer processes, it is extremely doubtful that equilibrium conditions can prevail at the interface; therefore, mass transfer transient effects would influence conditions at the interface, modifying the diffusive flux during the implosion, the condensation rate, and the peak temperatures and pressures reached during the last stages of the collapse.

In this work, the model proposed by Hauke et al. ${ }^{11}$ is used to determine the ranges of frequencies, bubble radius and forcing pressure amplitudes for which the influence of mass transfer effects is important. Typical values encountered in SBSL for amplitude and frequency of the driving pressure wave, as well as for the bubble initial radius are investigated. In practical applications, other variables like the gas composition might bear a direct impact upon chemical and physical processes near the interface; consequently, the analysis might become more intrincate. ${ }^{6}$

\section{MODEL}

The present analysis is based on the model of Hauke et al. ${ }^{11}$ where the continuity, momentum and energy conservation equations are solved inside a single bubble assuming spherical symmetry,

$$
\begin{aligned}
& \frac{D \rho}{D t}+\frac{\rho}{r^{2}} \frac{\partial\left(v_{r} r^{2}\right)}{\partial r}=0, \\
& \rho \frac{D Y_{\alpha}}{D t}=-\frac{1}{r^{2}} \frac{\partial}{\partial r}\left(r^{2} j_{\alpha}^{\mathrm{diff}}\right), \\
& \rho \frac{D v_{r}}{D t}=-\frac{\partial p}{\partial r}+\frac{1}{r^{2}} \frac{\partial}{\partial r}\left(r^{2} \tau_{r r}\right)-\frac{\tau_{\theta \theta}+\tau_{\phi \phi}}{r}, \\
& \rho \frac{D e}{D t}=-p(\nabla \cdot v)-\frac{1}{r^{2}} \frac{\partial\left(r^{2} q_{r}\right)}{\partial r}+\phi_{v},
\end{aligned}
$$

where $t$ stands for time, $r$ is the radial coordinate, $\rho$ is the density of the gas mixture, $v_{r}$ is the fluid radial velocity, $Y_{\alpha}$ and $j_{\alpha}^{\text {diff }}$ are the mass fraction and the diffusive mass flux, respectively, of species $\alpha, p$ is the pressure, $\tau_{r r}, \tau_{\theta \theta}$ and $\tau_{\phi \phi}$ are diagonal components of the viscous stress tensor, $e$ is the specific internal energy, $q_{r}$ is the radial heat flux and $\phi_{v}$ is the viscous dissipation function. Moreover, the temperature will be denoted by $T$.
For simplicity, and given that it provides realistic values for a wide range of pressures and temperatures,

$$
p_{g}=\rho_{g} R_{0} \sum_{\alpha=1}^{N} \frac{Y_{\alpha}}{W_{\alpha}} T_{g},
$$

where $R_{0}$ is the perfect gas universal constant, $W_{\alpha}$ is the molecular mass of species $\alpha$ and $p_{g}$ is the total gas pressure in the bubble, sum of all partial pressures (including that of the water vapor).

Radial and temporal dependence of all the variables is considered. The liquid continuity and momentum equations are replaced by the Rayleigh-Plesset equation, ${ }^{25}$ modified to account for compressibility effects ${ }^{26}$ and mass transfer processes ${ }^{27}$ this equation has been extensively used by other authors. ${ }^{28,29,12,27}$ The energy equations inside the bubble and in the liquid are coupled, avoiding the introduction of additional models for the determination of the interface temperature, which is assumed to be continuous. The species conservation equation [Eq. (2)] is solved inside the bubble in order to obtain the vapor concentration profile. The diffusion flux of gas across the interface is negligible along 1 cycle $^{6}$ and therefore, the species equation in the liquid is not used.

The Hertz-Knudsen-Langmuir formula, ${ }^{16,17}$ derived from kinetic theory of gases, is used to model mass transfer:

$$
J_{\mathrm{H}_{2} \mathrm{O}}^{\mathrm{tot}}=\frac{\beta\left(p_{\mathrm{sat}}-p_{\mathrm{H}_{2} \mathrm{O}}\right)}{\sqrt{2 \pi R^{0} T_{\mathrm{int}}}},
$$

where $J_{\mathrm{H}_{2} \mathrm{O}}^{\text {tot }}$ is the water evaporation/condensation flux across the interface, $p_{\mathrm{H}_{2} \mathrm{O}}$ is the partial water pressure at the interface, $R^{0}$ is the universal perfect gas constant, $T_{\text {int }}$ is the temperature at the interface and $p_{\text {sat }}$ is the equilibrium interface partial water pressure. Theoretically, equilibrium conditions are given by the Clausius-Clapeyron equation. However, it is possible to replace it by the experimental fitting of the equilibrium data using the Antoine equation:

$$
\ln \frac{p_{\text {sat }}}{133.322}=A_{k}-\frac{B_{k}}{\left(C_{k}+T_{\text {sat }}\right)} \text {. }
$$

In the particular case of water, the values of the constants are

\begin{tabular}{ccc}
$A_{k}$ & $B_{k}$ & $C_{k}$ \\
\hline 18.3036 & 3816.44 & -46.13
\end{tabular}

Equation (6) provides the total flux across the interface as a function of an empirical accommodation coefficient, $\beta$. Along this work, the same correlation as that in Refs. 11 and 15 is used to obtain the values of $\beta$,

$\beta= \begin{cases}0.35 & \text { if } T_{\mathrm{int}}<350 \mathrm{~K}, \\ 0.35-0.05 k^{(1)}-0.05 k^{(2)}+0.025 k^{(3)}, & \text { if } 350 \mathrm{~K} \leq T_{\mathrm{int}} \leq 500 \mathrm{~K}, \\ \frac{0.05}{T_{c}-500}\left(T_{c}-500\right) & \text { if } 500 \mathrm{~K} \leq T_{\mathrm{int}} \leq T_{c}, \\ 0, & T \geq T_{c},\end{cases}$ 


$$
k^{(m)}=k(k-1) \cdots[k-(m-1)]
$$

and

$$
k=\frac{T_{\text {int }}}{50}-7,
$$

where $T_{c}$ is the critical temperature.

For some predictions, the previous expressions are not used, and the value of beta is set equal to a constant predefined value.

\section{A. Boundary and initial conditions}

Appropriate boundary conditions are used to solve the governing differential equations. At the bubble center, spherical symmetry implies no radial gradients,

$$
\frac{\partial Y_{\alpha}}{\partial r}=\frac{\partial \rho_{g}}{\partial r}=\frac{\partial T_{g}}{\partial r}=\frac{\partial v_{g}}{\partial r}=0 \quad r=0, \quad \forall t .
$$

Moreover, the velocity at the center of the bubble is zero:

$$
v_{g}=0 \quad r=0, \quad \forall t .
$$

The continuity condition at the interface implies a relation among the gas velocity $v_{g}(R, t)$, the evaporation flux and the interface velocity $\dot{R}$, namely

$$
v_{g}(R, t)=\dot{R}-\frac{J_{\mathrm{H}_{2} \mathrm{O}}}{\rho_{g}} .
$$

It is assumed that water is the only compound with a net flux across the interface. The balance of water mass fraction at the interface relates $J_{\mathrm{H}_{2} \mathrm{O}}$ to the advective and diffusive fluxes,

$$
J_{\mathrm{H}_{2} \mathrm{O}}^{\mathrm{tot}}=\rho_{g}\left(v_{g}-\dot{R}\right) Y_{\mathrm{H}_{2} \mathrm{O}}^{g}-\rho_{g} D_{\alpha} \frac{\partial Y_{\mathrm{H}_{2} \mathrm{O}}^{g}}{\partial r},
$$

where $J_{\mathrm{H}_{2} \mathrm{O}}^{\text {tot }}$ is defined by Eq. (6).

An energy balance at the interface, $r=R(t)$, establishes a relation among the heat fluxes at the liquid and gas sides and the latent energy of evaporation,

$$
\kappa_{l} \frac{\partial T_{l}}{\partial r}=\kappa_{g} \frac{\partial T_{g}}{\partial r}+\Delta H_{\mathrm{vap}} J_{\mathrm{H}_{2} \mathrm{O}} .
$$

The variation of the enthalpy of vaporization with the temperature, $\Delta H_{\text {vap }}$, is calculated from the Watson equation. ${ }^{30}$

The numerical procedure assumes a continuous temperature profile across the interface.

$$
T_{g}=T_{l} .
$$

In order to solve the energy equation, a constant liquid temperature far away from the bubble is imposed,

$$
T_{l}=T_{\infty} \quad(r \rightarrow \infty) .
$$

A bubble in mechanical and thermodynamic equilibrium with its surrounding liquid is initially assumed. Reference pressure and temperature are employed to calculate all properties from equilibrium balances. A unique insoluble gas would be considered here; thus, the initial vapor content is taken as uniform and equal to the saturation condition.
TABLE I. Characteristic parameters.

\begin{tabular}{lc}
\hline \hline$T_{\text {liq }}$ & $295 \mathrm{~K}$ \\
$P_{\text {liq }}$ & $101300 \mathrm{~Pa}$ \\
$P_{\text {vap }}$ & $2500 \mathrm{~Pa}$ \\
$\mu_{l}$ & $8 \times 10^{-4} \mathrm{~kg} / \mathrm{m} \mathrm{s}$ \\
$\rho_{l}$ & $1000 \mathrm{~kg} / \mathrm{m}^{3}$ \\
$c_{l}$ & $1500 \mathrm{~m} / \mathrm{s}$ \\
$\sigma$ & $0.06 \mathrm{~N} / \mathrm{m}$ \\
$\Delta P$ & $100000 \mathrm{~Pa}$ \\
\hline \hline
\end{tabular}

This system of nonlinear partial differential equations is solved with an Arbitrary Lagrangian-Eulerian ${ }^{31}$ (ALE) finite element method combined with a 5/6th-order Fehlberg Runge-Kutta method. ${ }^{32}$ Further details about the physical model and numerical method can be found in Ref. 11 .

\section{RESULTS}

This study is focused on typical SBSL conditions. ${ }^{33} \mathrm{~A}$ single bubble with an initial radius of $20 \mu \mathrm{m}$ is immersed in an ultrasonic field; the reference case parameters are specified in Table I. The amplitude and frequency of the driving pressure wave, as well as the bubble initial radius, will be varied to analyze mass transfer effects on bubble evolution.

The presence of Argon, a non-condensable gas, is assumed. This implies that, in addition to non-equilibrium effects, the water vapor diffusion in Argon plays a critical role in mass transfer processes. ${ }^{9}$

The maximum temperatures and pressures as a function of the forcing frequency are plotted in Fig. 1 for $\Delta P$ $=0.9 \mathrm{~atm}$ and $\Delta P=1 \mathrm{~atm}$. The values of the frequencies are made dimensionless by using the bubble natural frequency defined by

$$
\omega_{\mathrm{n}}=\sqrt{\frac{3 \gamma p_{\infty}}{\rho_{l} R_{b}^{2}}},
$$

where $\gamma$ is the specific heat ratio and $p_{\infty}$ is the reference pressure. When the minimum pressure in the liquid does not reach its vapor pressure $(\Delta P \leq 0.9 \mathrm{~atm})$ no significant changes in peak pressures and temperatures are observed with and without mass transfer at any frequency.

For a pressure amplitude $\Delta P=1 \mathrm{~atm}$, the sensitivity of implosions to mass transfer becomes apparent at forcing frequencies below about $30 \mathrm{kHz}$. Both, peak pressures and temperatures increase as mass transfer effects are taken into account. The intensity of implosions is considerably enhanced due to mass transfer, this trend being more marked at lower frequencies. This result is encountered for a wide range of bubble radii even for the relative small amplitudes tested in this work. Figure 2 depicts the differences between peak pressures obtained taking into account mass transfer and those using $\beta=0$ as a function of dimensionless frequency. The parameter $\epsilon_{T}$ is a dimensionless measure of the difference of peak temperatures with and without mass transfer, 

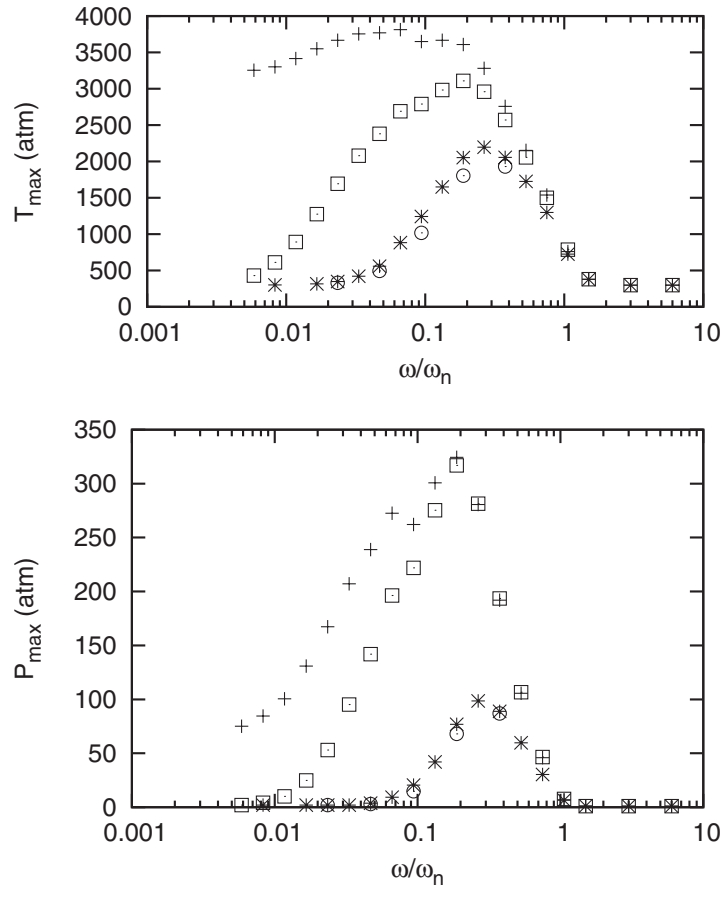

$\Delta P=1 \operatorname{atm}(\beta=0.5)$

$\Delta P=1 \mathrm{~atm}(\beta=0)$

$\Delta P=0.9$ atm $(\beta=0.5) \quad *$

$\Delta P=0.9$ atm $(\beta=0)$

FIG. 1. Peak pressures and temperatures for an argon-vapor bubble of $20 \mu \mathrm{m}$ as a function of the dimensionless frequency for two different pressure wave amplitudes. Mass transfer effects have a significant influence on the peak temperatures and pressures for $\Delta P \geq 1$ atm and frequencies below $30000 \mathrm{~Hz}$.

$$
\epsilon_{T}=\frac{T_{\max }(\beta=0.5)-T_{\max }(\beta=0)}{T_{\max }(\beta=0.5)} .
$$

In general, the larger the bubble, the larger the area of mass exchange and the higher the sensitivity to the computed mass transfer. Moreover, the range of frequencies for which significant differences with and without mass transfer are observed becomes wider for larger bubbles.

The effect of mass transfer on bubble dynamics and, in particular, on its radius evolution, is small for frequencies greater than $250 \mathrm{kHz}$, irrespective of the value of $\Delta P$. In any

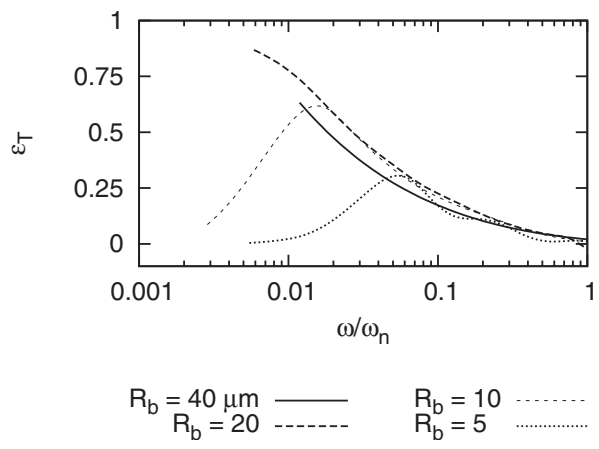

FIG. 2. Influence of the mass transfer model on the peak temperatures during the implosion for different bubble radius and $\Delta P=1$ atm (definition of $\epsilon_{T}$ contained in the text). The effect of mass transfer is especially important for large bubbles (of the order of $40 \mu \mathrm{m}$ ) and low frequencies even for relative low amplitudes of excitation.
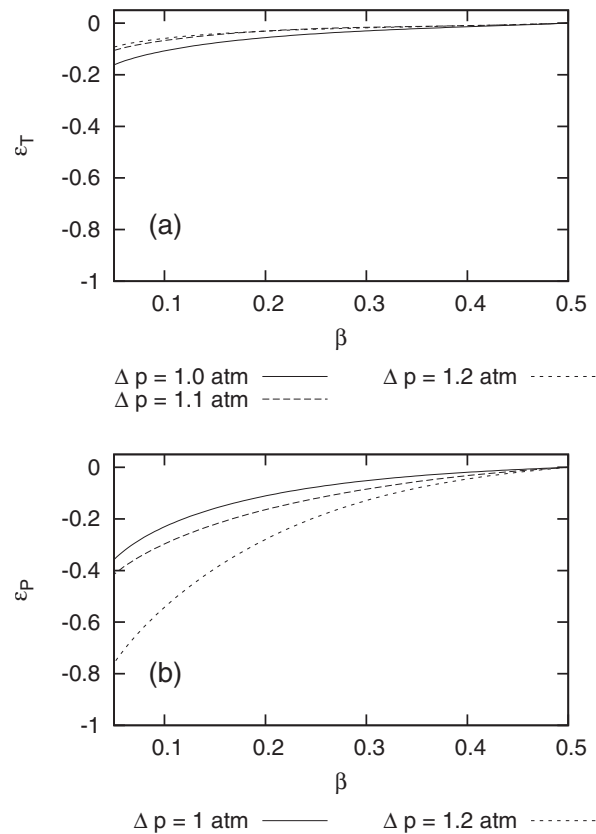

$$
\begin{array}{r}
\Delta \mathrm{p}=1 \mathrm{~atm} \\
\Delta \mathrm{p}=1.1 \mathrm{~atm}
\end{array}
$$

FIG. 3. Influence of the mass transfer on the peak temperatures and pressures as functions of $\beta$, for a single cavitating bubble of $20 \mu \mathrm{m}$ in an ultrasonic field of $5 \mathrm{kHz}$, and for different driving pressure wave amplitudes. Peak pressures are especially sensitive to the values of beta for large $\Delta P$.

event, the value of $\beta$ is essential to accurately predict some internal processes taking place inside the bubble, such as radical formation. ${ }^{10,11}$

In order to gain more insight into the physical mechanisms responsible for differences in peak pressures and temperatures, a bubble of initial radius of $20 \mu \mathrm{m}$ subjected to an ultrasonic field of $5000 \mathrm{~Hz}$ is analyzed. Figure 3 depicts peak temperatures and pressures as functions of $\beta$, for different values of the forcing pressure wave amplitude. The definition of $\epsilon_{P}$ is identical to that of $\epsilon_{T}$ in Eq. (19), changing $T_{\max }$ by $P_{\max }$. Peak pressures turn out to be especially sensitive to the value of $\beta$, particularly for large pressure wave amplitudes. In this case, neglecting mass transfer effects is no justifiable, because of the very low pressures attained inside the bubble during the expansion should evaporation be considered. This fact is apparent in Fig. 4, where bubble radius, vapor concentration at the bubble interface, and pressure evolutions are plotted for an initial radius of $20 \mu \mathrm{m}$, in an ultrasonic field of $5000 \mathrm{~Hz}$ and for $\Delta P=1.1$ atm. The pressure temporal evolution indicates that, irrespective of the value of the accommodation coefficient, the evaporation flux during the expansion prevents attaining the low pressures predicted by the model in the absence of mass transfer; this feature has an important influence on the predicted temperatures and pressures during the collapse.

The effect of mass transfer processes on the bubble dynamics can be assessed utilizing the dimensionless evaporation flux, $J^{\text {tot }} /\left(\rho_{b}(R) \dot{R}\right)$. This parameter is large when mass transfer has an important influence on the interface velocity, whereas it tends to zero when the evaporation can be neglected. Figure 5 depicts the temporal radius evolution as well as the dimensionless evaporation flux. $J^{\text {tot } /\left(\rho_{b}(R) \dot{R}\right)}$ spans over several orders of magnitude and its absolute value 

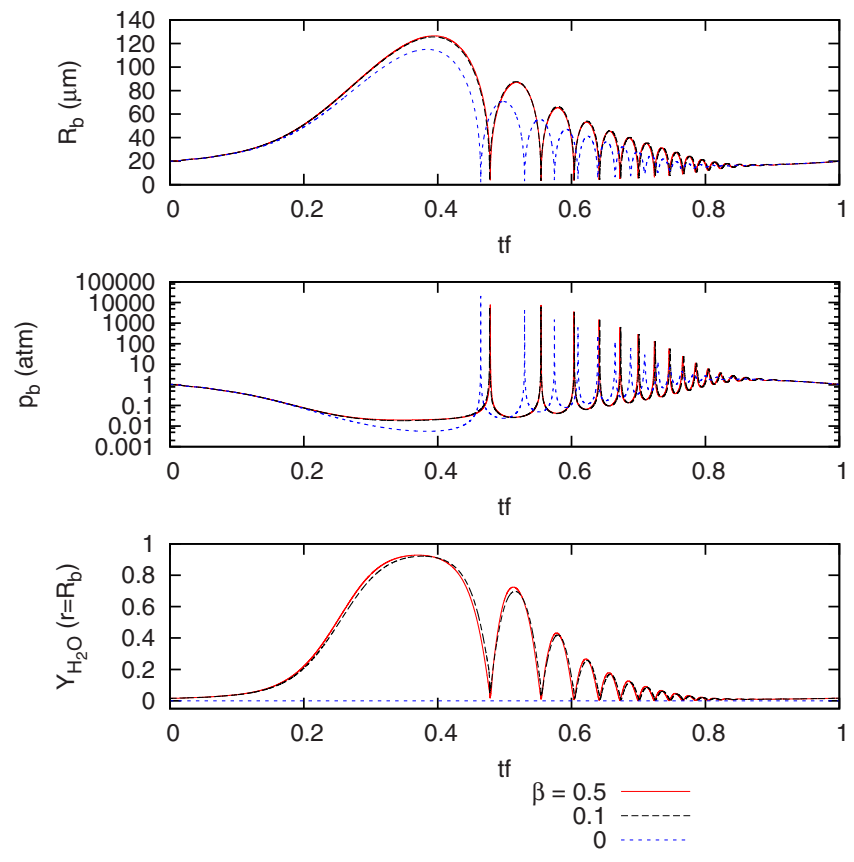

FIG. 4. (Color online) Temporal evolutions of the bubble radius, bubble pressure at $r=0$ and vapor mass fraction at the interface for an initial radius of $20 \mu \mathrm{m}$, in an ultrasonic field of $5000 \mathrm{~Hz}$ and $\Delta P=1.1$ atm. Whereas differences of the maximum radius reached at the end of the expansion are small, large differences in peak pressures are observed for different values of $\beta$.

is plotted. During the compression, condensation occurs and $J^{\text {tot }} /\left(\rho_{b}(R) \dot{R}\right)$ is negative, whereas the opposite situation is encountered during the expansion. It is important to emphasize that the effect of mass transfer processes can be reflected by the absolute value, independently on whether evaporation or condensation occurs.

Mass transfer has an important influence on the interface velocities, and, therefore, on the maximum bubble radius during the expansion stage. However, although the maximum radius is influenced by the mass transfer, the sensitivity to the
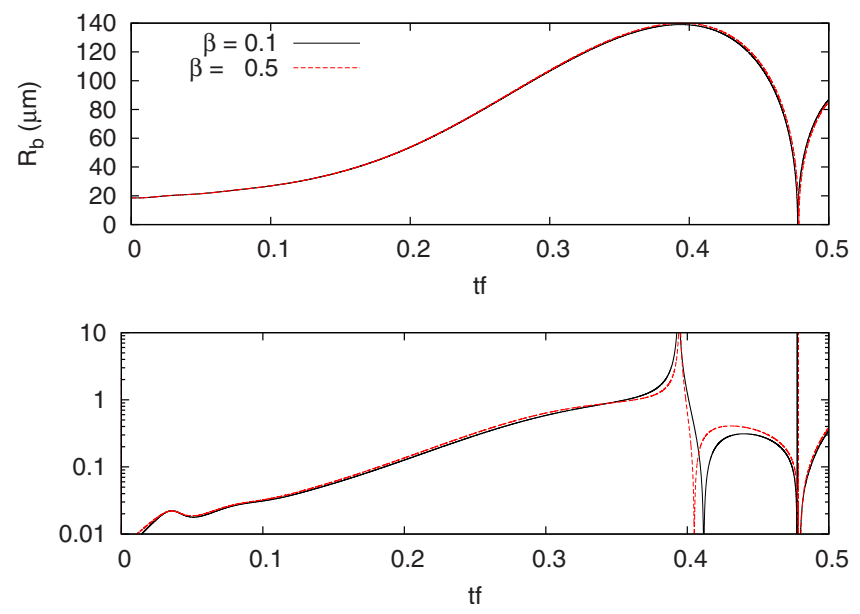

FIG. 5. (Color online) Bubble radius and dimensionless evaporation flux, $J^{\text {tot }} /\left(\rho_{b}(R) \dot{R}\right)$, temporal evolutions for two different values of $\beta$. Parameter values are the same than in Fig. 4. The interface velocity during the expansion is only moderately influenced by mass transfer. On the other hand, compression velocities are significantly affected by mass transfer and are very sensitive to the value of $\beta$. value of $\beta$ of the maximum radius and the water vapor content is small. Equilibrium conditions are encountered at the interface at every instant and thus, transient effects do not influence the vapor content of the bubble during the expansion. On the other hand, the characteristic expansion velocities are usually small and then, large pressure wave amplitudes are required in order to observe significant changes of the maximum radius. This observation is in agreement with Ref. 2, where large amplitudes are suggested in order to observe significant differences of the maximum bubble radius attained during the expansion.

During the compression stage, mass transfer has also an important effect on the predicted velocities. In this case, unlike during the expansion stage, the compression velocities and the values of $J^{\text {tot }} /\left(\rho_{b}(R) \dot{R}\right)$ are highly sensitive to the value of $\beta$ (Fig. 5). Thus, any parameter influencing this velocity, has a direct influence on peak temperatures and pressures. The effect of $\beta$ on peak pressures is more pronounced than that on peak temperatures (Fig. 5), because the former are strongly determined by compression velocities, whereas the latter are also influenced by heat exchange processes across the interface. Note that although the increase of water vapor content lowers the peak temperatures during the collapse, ${ }^{4}$ this effect is unrelated to the value of the accommodation coefficient because the vapor content reached during the expansion is not influenced by $\beta$. It should also be emphasized that the influence of $\beta$ on the compression velocities is encountered during the initial stage of the compression, when the conditions inside the bubble are not extreme and the model used here is expected to provide reasonably accurate results. Therefore, although peak pressures and temperatures could still be influenced by some effects not included in the present model, it is not the case of the compression velocities reached during the first stage of the collapse. This behavior is encountered for frequencies in the range 1000-10000 Hz, regime in which further studies could provide some new insight about realistic values of the accommodation coefficient.

\section{CONCLUSIONS}

This study emphasizes and proves the importance of correctly modeling mass transfer processes for predicting the single bubble response in SBSL experiments. In general, evaporation/condensation processes across the interface have been shown to significantly modify the bubble dynamics. As a first approximation, mass transfer can be neglected for frequencies greater than $250 \mathrm{kHz}$.

Mass transfer processes typically enhance both expansion and collapse stages. When the amplitude of the pressure wave is large enough (namely, larger than 1 atm for the conditions investigated in the present work), evaporation/ condensation has an important effect on bubble implosions. The value of the accommodation coefficient then plays a crucial role on the corresponding model results; depending on its value, strong implosions can be predicted for a relatively broad range of frequencies (one or two orders of magnitude lower than those with no mass transfer). These differ- 
ent behaviors are only observed for large driving pressure amplitudes, being more pronounced for large bubbles and low frequencies.

The temporal evolution of bubbles radius with initial values of a few tens of microns undergoing an ultrasonic field with frequencies in the range from 1 to $10 \mathrm{kHz}$ might provide hints to estimate a practical and realistic value of the accommodation coefficient, as proposed by Puente and Boneto. ${ }^{24}$ Mass transfer processes might only moderately effect the maximum radius attained during the expansion; however, they can have an influence on the peak temperatures and pressures reached during the implosion.

In summary, it can be concluded that the correct simulation of mass transfer processes in SBSL is especially important for large pressure wave amplitudes and for frequencies in a range going from the resonant frequency to frequencies one or two orders of magnitude smaller.

\section{ACKNOWLEDGMENTS}

The authors would like to acknowledge the comments and suggestions of Dr. Jean-Louis Thomas on a previous version of this manuscript. This work has been partially supported by Ministerio de Ciencia y Tecnologia of Spain, under Grant No CTM2004-06184-C02-02.

${ }^{1}$ Y. Hao and A. Prosperetti, "The dynamics of vapor bubbles in acoustic pressure fields," Phys. Fluids 11, 2008-2019 (1999).

${ }^{2}$ A. T. Preston, T. Colonius, and C. E. Brennen, "A reduced order model of diffusive effects on the dynamics of bubbles," Phys. Fluids 19, 123302 (2007).

${ }^{3}$ M. P. Brenner, S. Hilgenfeldt, and D. Lohse, "Single-bubble sonoluminiscence," Rev. Mod. Phys. 74, 425-484 (2002).

${ }^{4}$ R. Toegel, B. Gompf, R. Pecha, and D. Lohse, "Does water vapor prevent upscaling sonoluminiscence?,” Phys. Rev. Lett. 85, 3165-3168 (2000).

${ }^{5}$ S. Hilgenfeldt and D. Lohse, "Predictions for upscaling sonoluminiscence," Phys. Rev. Lett. 82, 1036-1039 (1999).

${ }^{6} \mathrm{R}$. Toegel and D. Lohse, "Phase diagrams for sonoluminescing bubbles: A comparison between experiment and theory," J. Chem. Phys. 118, 18631875 (2003).

${ }^{7}$ S. Hilgenfeldt, D. Lohse, and M. P. Brenner, "Phase diagrams for sonoluminescing bubbles," Phys. Fluids 8, 2808-2826 (1996).

${ }^{8}$ S. Sochard, A. M. Wilhelm, and H. Delmas, "Modelling of free radicals production in a collapsing gas-vapour bubble," Ultrason. Sonochem. 4, 77-84 (1997)

${ }^{9}$ B. D. Storey and A. J. Szeri, "Water vapour, sonoluminiscence and sonochemistry," Proc. R. Soc. London, Ser. A 456, 1685-1709 (2000).

${ }^{10}$ A. J. Colussi and M. R. Hoffmann, "Vapor supersaturation in collapsing bubbles. relevance to the mechanisms of sonochemistry and sonoluminiscence," J. Phys. Chem. A 103, 11336-11339 (1999).

${ }^{11}$ G. Hauke, D. Fuster, and C. Dopazo, "Dynamics of a single cavitating and reacting bubble," Phys. Rev. E 75, 066310 (2007).

${ }^{12}$ N. Xu, R. E. Apfel, A. Khong, X. Hu, and L. Wang, "Water vapor diffusion effects on gas dynamics in a sonoluminescing bubble," Phys. Rev. E 68, 016309 (2003).

${ }^{13}$ Y. An and C. Ying, "Model of single bubble sonoluminiscence," Phys. Rev. E 71, 036308 (2005)

${ }^{14}$ T. Ishiyama, T. Yano, and S. Fujikawa, "Molecular dynamics study of kinetic boundary condition at an interface between a polyatomic vapor and its condensed phase," Phys. Fluids 16, 4713-4726 (2004).

${ }^{15} \mathrm{~K}$. Yasui, "Alternative model of single sonoluminiscence," Phys. Rev. E 56, 6750-6760 (1997).

${ }^{16} \mathrm{H}$. Hertz, "Über die verdunstug der flüssigkeiten, inbesondere des quecksilbers im lufteren räume (On the evaporation of fluids, especially of mercury, in vacuum spaces)," Ann. Phys. 17, 177 (1982).

${ }^{17}$ M. Knudsen, "Maximum rate of vaporization of mercury," Ann. Phys. 352, 697-708 (1915).

${ }^{18}$ I. W. Eames, N. J. Marr, and H. Sabir, "The evaporation coefficient of water: A review," Int. J. Heat Mass Transfer 40, 2963-2973 (1997).

${ }^{19}$ N. A. Gumerov, C. T. Hsiao, and A. G. Goumilevski, "Determination of the accommodation coefficient using vapor/gas bubble dynamics in an acoustic field," Technical Report No. 1, Dynaflow, Inc., Fulton, MD, 2001; see also http://gltrs.grc.nasa.gov/GLTRS (Last viewed 09/05/2004).

${ }^{20} \mathrm{~J}$. Barrett and C. Clement, "Kinetic evaporation and condensation rates and their coefficients," J. Colloid Interface Sci. 150, 352-364 (1992).

${ }^{21}$ D. E. Hagen, J. Schmitt, M. Trueblood, J. Carstens, D. R. White, and D. J. Alofs, "Condensation coefficient measurement for water in the UMR cloud simulation chamber," J. Atmos. Sci. 46, 803-816 (1989).

${ }^{22}$ U. Narusawa and G. S. Springer, "Measurements of evaporation rates of water," J. Colloid Interface Sci. 50, 392-395 (1975).

${ }^{23}$ M. Volmer, "Kinetik der Phasebildung (Kinitics of phase change)," (Steinkopff, Leipzig, 1939).

${ }^{24}$ G. F. Puente and F. J. Bonetto, "Proposed method to estimate the liquidvapor accommodation coefficient based on experimental sonoluminescence data," Phys. Rev. E 71, 056309 (2005).

${ }^{25} \mathrm{~J}$. W. Strutt, "On the pressure developed in a liquid during the collapse of a spherical cavity," Philos. Mag. 34, 94-98 (1917).

${ }^{26} \mathrm{~J}$. Keller and M. Miksis, "Bubble oscillations of large amplitude," J. Acoust. Soc. Am. 68, 628-633 (1980).

${ }^{27}$ K. Yasui, T. Tuziuti, M. Sivakumar, and Y. Iida, "Theoretical study of single-bubble sonochemistry," J. Chem. Phys. 122, 224706 (2005).

${ }^{28}$ H. Lin, B. D. Storey, and A. J. Szeri, "Inertially driven inhomogeneities in violently collapsing bubbles: The validity of the Rayleigh-Plesset equation," J. Fluid Mech. 452, 145-162 (2002).

${ }^{29}$ B. D. Storey and A. J. Szeri, "Argon rectification and the cause of light emission in single-bubble sonoluminiscence," Phys. Rev. Lett. 88, 074301 (2002).

${ }^{30}$ G. Narsimhan, Br. Chem. Eng. 10, 253 (1965).

${ }^{31}$ T. J. R. Hughes, W. K. Liu, and T. K. Zimmerman, "Langrangian-Eulerian finite element formulation for incompressible viscous flows," Comput. Methods Appl. Mech. Eng. 29, 329-349 (1981).

${ }^{32}$ W. H. Press, S. A. Teulkolsky, W. T. Vetterling, and B. P. Flannery, $N u-$ merical Recipes in Fortran 77 (Cambridge University Press, Cambridge, 1992).

${ }^{33}$ F. Gaitan, L. A. Crum, C. Church, and R. Roy, "Sonoluminiscence and bubble dynamics for a single, stable, cavitation bubble," J. Acoust. Soc. Am. 91, 3166-3183 (1992). 